\title{
Global properties of the spectrum of the Haldane-Shastry spin chain
}

\author{
Federico Finkel* and Artemio González-López ${ }^{\dagger}$ \\ Departamento de Física Teórica II, Universidad Complutense, 28040 Madrid, Spain \\ (Received 22 July 2005; revised manuscript received 1 September 2005; published 8 November 2005)
}

\begin{abstract}
We derive an exact expression for the partition function of the $\mathrm{su}(m)$ Haldane-Shastry spin chain, which we use to study the density of levels and the distribution of the spacing between consecutive levels. Our computations show that when the number of sites $N$ is large enough, the level density is Gaussian to a very high degree of approximation. More surprisingly, we also find that the nearest-neighbor spacing distribution is not Poissonian, so that this model departs from the typical behavior for an integrable system. We show that the cumulative spacing distribution of the model can be well approximated by a simple functional law involving only three parameters.
\end{abstract}

DOI: $10.1103 /$ PhysRevB.72.174411

PACS number(s): 75.10.Pq, 05.30.-d, 05.45.Mt

\section{INTRODUCTION}

The Haldane-Shastry (HS) spin chain describes $N$ spins equally spaced on a circle with an interaction inversely proportional to the square of their chord distance. ${ }^{1,2}$ The original motivation for studying this model is the fact that it possesses an exact Jastrow-product ground state, which coincides with the $U \rightarrow \infty$ limit of Gutzwiller's variational wave function for the Hubbard model, ${ }^{3-5}$ and also with the onedimensional version of the resonating valence bond state introduced by Anderson. ${ }^{6}$ Since its very introduction, the HS spin chain has been extensively studied as a completely integrable model ${ }^{7}$ solvable by the asymptotic Bethe ansatz, ${ }^{8-10}$ whose spinon excitations provide a simple example of a system obeying fractional statistics. ${ }^{11}$

The energy spectrum of the HS Hamiltonian with spin $1 / 2$ was partially computed in the original papers of Haldane and Shastry. In a subsequent publication, ${ }^{12}$ Haldane et al. empirically found a complete description of the spectrum for arbitrary spin and explained its highly degenerate character by the symmetry of the model under the Yangian algebra $\mathcal{Y}\left(\mathrm{sl}_{m}\right)$. These results were rigorously established in Ref. 13 by explicitly constructing a transfer matrix in terms of the Dunkl operators ${ }^{14,15}$ of the trigonometric Sutherland dynamical model. ${ }^{16,17}$ In this approach, the spectrum is obtained by considering all possible motifs $\left.\delta \equiv\left(0 \delta_{1} \cdots \delta_{N-1}\right)\right)$, where each $\delta_{j}$ is either 0 or 1 and the maximum number of consecutive 1 's is $m-1$. Indeed, the energy associated with a motif $\delta$ is given by the compact formula

$$
E_{\mathrm{HS}}(\delta)=\sum_{j=1}^{N-1} \delta_{j} j(j-N)
$$

The degeneracy of a level $E_{\mathrm{HS}}$ is obtained by summing the degeneracies corresponding to all the motifs $\delta$ such that $E_{\mathrm{HS}}(\delta)=E_{\mathrm{HS}}$. Although there is a well-defined algorithm for computing the degeneracy of each motif, in practice the computation becomes quite involved except for $m=2$. Therefore, it is difficult to derive in this way an exact expression for the partition function valid for arbitrary values of $N$ and $m$. Perhaps as a consequence of this fact, little attention has been paid in the literature to the global properties of the spectrum of the HS chain.

Some authors ${ }^{18,19}$ have suggested that the main obstacle in computing the partition function of the HS chain in closed form is the fact that the dispersion relation (1) is nonlinear in $j$, in contrast with the Polychronakos rational chain. ${ }^{20,21}$ In a recent paper, ${ }^{22}$ however, the partition function of the trigonometric HS spin chain of $B C_{N}$ type has been exactly computed applying what is known as Polychronakos's freezing trick, ${ }^{23}$ notwithstanding the fact that these chains have a nonlinear dispersion relation similar to (1). In fact, we shall prove in what follows that the partition function of the chain (2) can also be computed using the freezing trick. From the partition function, it is straightforward to generate the spectrum of the HS chain for a wide range of values of $N$ and $m$ and, thus, study global properties thereof, such as the level density or the distribution of the spacing between consecutive levels.

\section{PARTITION FUNCTION}

For convenience, we shall take the Hamiltonian of the (antiferromagnetic) Haldane-Shastry spin chain as

$$
H=\frac{1}{2} \sum_{i<j} \sin \left(\xi_{i}-\xi_{j}\right)^{-2}\left(1+S_{i j}\right),
$$

where $\xi_{i}=i \pi / N$ and $S_{i j}$ is the spin permutation operator of particles $i$ and $j$. Here and throughout the paper, all sums and products run from 1 to $N$ unless otherwise specified. The Hamiltonian of the original HS spin chain is given by $H_{\mathrm{HS}}$ $=H-E_{\max }$, where

$$
E_{\max } \equiv \sum_{i<j} \sin \left(\xi_{i}-\xi_{j}\right)^{-2}
$$

is the highest energy of $H$. In order to apply the freezing trick, we need to introduce the Sutherland spin model

$$
H^{*}=-\sum_{i} \partial_{x_{i}}^{2}+a \sum_{i \neq j} \sin \left(x_{i}-x_{j}\right)^{-2}\left(a+S_{i j}\right),
$$

and its scalar version 


$$
H_{0}=-\sum_{i} \partial_{x_{i}}^{2}+a(a-1) \sum_{i \neq j} \sin \left(x_{i}-x_{j}\right)^{-2} .
$$

We thus have

$$
H^{*}=H_{0}+4 a \mathbf{H},
$$

where $\mathbf{H}$ is obtained from $H$ by the replacement $\xi_{i} \rightarrow x_{i}$. The freezing trick is based on the fact that for $a \rightarrow \infty$ the particles "freeze" at the equilibrium positions of the scalar part of the potential in $H^{*}$, which are simply the lattice points of the chain (2). In this limit, the spin degrees of freedom decouple from the dynamical ones, so that, by Eq. (5), the energies of the dynamical spin model are approximately given by ${ }^{22}$

$$
E_{i j}^{*} \simeq E_{0, i}+4 a E_{j},
$$

where $E_{0, i}$ and $E_{j}$ are any two levels of $H_{0}$ and $H$. Hence, the partition functions $Z, Z^{*}$, and $Z_{0}$ of $H, H^{*}$, and $H_{0}$, respectively, satisfy the approximate equality

$$
Z^{*}(T) \simeq Z_{0}(T) Z\left(\frac{T}{4 a}\right), \quad a \gg 1 .
$$

The latter equation leads to the exact formula

$$
Z(T)=\lim _{a \rightarrow \infty} \frac{Z^{*}(4 a T)}{Z_{0}(4 a T)},
$$

which we will use to compute the partition function of the chain (2) in closed form.

In order to evaluate the right-hand side (RHS) of (7), we need to compute the spectra of $H^{*}$ and of its scalar limit $H_{0}$. These spectra can be obtained in a unified way by considering the scalar differential-difference operator

$$
\bar{H}=-\sum_{i} \partial_{x_{i}}^{2}+a \sum_{i \neq j} \sin \left(x_{i}-x_{j}\right)^{-2}\left(a-P_{i j}\right),
$$

where $P_{i j}$ permutes the coordinates $i$ and $j$. The operator $\bar{H}$ is represented by an upper triangular matrix in a (nonorthonormal) basis whose elements are of the form

$$
\phi_{\mathbf{p}}(\mathbf{x})=e^{2 \mathbf{i p} \cdot \mathbf{x}} \prod_{i<j} \sin ^{a}\left(x_{i}-x_{j}\right),
$$

where the vector $\mathbf{p}=\left(p_{1}, \ldots, p_{N}\right) \in \mathbb{R}^{N}$ is such that the differences $p_{i}-p_{i+1}, 1 \leqslant i \leqslant N-1$, are integers. The basis elements (9) should be ordered in a suitable way that we shall now describe. We shall say that a vector $\hat{\mathbf{p}}=\left(\hat{p}_{1}, \ldots, \hat{p}_{N}\right)$ is nonincreasing if $\hat{p}_{i+1} \leqslant \hat{p}_{i}$ for $i=1, \ldots, N-1$. Given two nonincreasing vectors $\hat{\mathbf{p}}$ and $\hat{\mathbf{p}}^{\prime}$, we shall write $\hat{\mathbf{p}}<\hat{\mathbf{p}}^{\prime}$ if $\hat{p}_{1}-\hat{p}_{1}^{\prime}$ $=\cdots=\hat{p}_{i-1}-\hat{p}_{i-1}^{\prime}=0$ and $\hat{p}_{i}<\hat{p}_{i}^{\prime}$. Finally, we say that the basis element $\phi_{\mathbf{p}}$ precedes $\phi_{\mathbf{p}^{\prime}}$ if $\hat{\mathbf{p}}<\hat{\mathbf{p}}^{\prime}$, where $\hat{\mathbf{p}}$ and $\hat{\mathbf{p}}^{\prime}$ are the unique nonincreasing vectors obtained from $\mathbf{p}$ and $\mathbf{p}^{\prime}$ by reordering their components. It can then be shown that the matrix of $\bar{H}$ in the basis $\left\{\phi_{\mathbf{p}}\right\}$ with the order just defined is indeed upper triangular, with diagonal elements ${ }^{13,24}$

$$
\bar{E}(\mathbf{p})=\sum_{i}\left(2 \hat{p}_{i}+a(N+1-2 i)\right)^{2} .
$$

We shall now see how the spectrum of $H^{*}$ follows easily from that of $\bar{H}$. To this end, let us introduce the total anti- symmetrizer $\Lambda$ with respect to simultaneous permutations of the spatial and spin coordinates. We can construct a (nonorthonormal) basis of the Hilbert space of the Hamiltonian $H^{*}$ with states of the form

$$
\psi_{\mathbf{p}, \mathbf{s}}(\mathbf{x})=\Lambda\left(\phi_{\mathbf{p}}(\mathbf{x})|\mathbf{s}\rangle\right),
$$

where $|\mathbf{s}\rangle \equiv\left|\mathrm{s}_{1}, \ldots, \mathrm{s}_{N}\right\rangle$ is an element of the spin basis and the vector $\mathbf{p}$ satisfies the following conditions:

(i) The differences $n_{i} \equiv p_{i}-p_{i+1}, 1 \leqslant i \leqslant N-1$, are nonnegative integers.

(ii) At most $m$ components of $\mathbf{p}$ can be equal.

(iii) The total momentum vanishes, i.e., $\Sigma_{i} p_{i}=0$.

The first two conditions are a direct consequence of the antisymmetric nature of the states (11). The last condition reflects the fact that, since $H^{*}$ is translationally invariant, we can work in the center of mass frame. The basic states $\psi_{\mathbf{p}, \mathbf{s}}$ should be ordered in such a way that $\psi_{\mathbf{p}, \mathbf{s}}$ precedes $\psi_{\mathbf{p}^{\prime}, \mathbf{s}^{\prime}}$ if $\mathbf{p}<\mathbf{p}^{\prime}$ [note that the vectors $\mathbf{p}$ and $\mathbf{p}^{\prime}$ are nonincreasing by condition (i)].

From the elementary relations $P_{i j} \Lambda=-S_{i j} \Lambda$ and the fact that $\bar{H}$ clearly commutes with $\Lambda$, it follows that

$$
\begin{aligned}
H^{*} \psi_{\mathbf{p}, \mathbf{s}}= & \bar{H} \psi_{\mathbf{p}, \mathbf{s}}=\Lambda\left(\left(\bar{H} \phi_{\mathbf{p}}\right)|\mathbf{s}\rangle\right)=\Lambda\left(\bar{E}(\mathbf{p}) \phi_{\mathbf{p}}|\mathbf{s}\rangle\right. \\
& \left.+\sum_{\mathbf{p}^{\prime}<\mathbf{p}} c_{\mathbf{p p}^{\prime}} \phi_{\mathbf{p}^{\prime}}|\mathbf{s}\rangle\right)=\bar{E}(\mathbf{p}) \psi_{\mathbf{p}, \mathbf{s}}+\sum_{\mathbf{p}^{\prime}<\mathbf{p}} c_{\mathbf{p p}^{\prime}} \psi_{\mathbf{p}^{\prime}, \mathbf{s}} .
\end{aligned}
$$

Hence, the Hamiltonian $H^{*}$ of the Sutherland spin model is upper triangular in the basis $\left\{\psi_{\mathbf{p}, \mathbf{s}}\right\}$, with diagonal elements

$$
E^{*}(\mathbf{p}, \mathbf{s})=\sum_{i}\left(2 p_{i}+a(N+1-2 i)\right)^{2},
$$

where $\mathbf{p}$ satisfies conditions (i)-(iii) above.

The spectrum of $H_{0}$ can be derived by a similar argument, noting that $H_{0}=\bar{H}$ on scalar symmetric states of the form $\psi_{\mathbf{p}}=\Lambda_{s} \phi_{\mathbf{p}}$, where $\Lambda_{s}$ is the symmetrizer with respect to the spatial coordinates and $\mathbf{p}$ satisfies only conditions (i) and (iii) above. Hence, ${ }^{17}$ the eigenvalues $E_{0}(\mathbf{p})$ of $H_{0}$ are also given by the RHS of (12), where now $\mathbf{p}$ is not restricted by condition (ii).

From the above results it is easy to compute the partition functions $Z_{0}(4 a T)$ and $Z^{*}(4 a T)$ in the limit $a \rightarrow \infty$. For the computation of $Z_{0}(4 a T)$, we start by expanding the eigenvalues of $H_{0}$ in powers of $a$ as

$$
E_{0}(\mathbf{p})=a^{2} E^{0}+4 a \sum_{i}(N+1-2 i) p_{i}+O(1),
$$

where

$$
E^{0}=\sum_{i}(N+1-2 i)^{2}=\frac{1}{3} N\left(N^{2}-1\right) .
$$

Since $E^{0}$ does not depend on $\mathbf{p}$ and, therefore, contributes the same overall constant factor to both $Z_{0}$ and $Z^{*}$, we shall, henceforth, drop the first term in Eq. (13). With this convention, for $a \gg 1$ the denominator in Eq. (7) is given by 


$$
Z_{0}(4 a T) \simeq \sum_{\mathbf{p}} q^{\Sigma_{i} p_{i}(N+1-2 i)},
$$

where $q=e^{-1 /\left(k_{\mathrm{B}} T\right)}$ and the outer sum runs over all vectors $\mathbf{p}$ satisfying conditions (i) and (iii) above. Setting $n_{N} \equiv p_{N}$, we have

$$
\sum_{i} p_{i}(N+1-2 i)=\sum_{j \geqslant i} n_{j}(N+1-2 i)=\sum_{j=1}^{N-1} j(N-j) n_{j} .
$$

Taking into account that $n_{N}$ is determined by the remaining $n_{i}$ 's by condition (iii), we finally obtain

$$
Z_{0}(4 a T) \simeq \sum_{n_{1}, \ldots, n_{N-1} \geqslant 0} \prod_{j=1}^{N-1} q^{j(N-j) n_{j}}=\prod_{j=1}^{N-1}\left(1-q^{j(N-j)}\right)^{-1} .
$$

In order to compute the partition function $Z^{*}(4 a T)$ for $a \gg 1$, it is convenient to represent the vector $\mathbf{p}$ labeling the energies (12) of $H^{*}$ as

$$
\mathbf{p}=(\overbrace{\rho_{1}, \ldots, \rho_{1}}^{k_{1}}, \ldots, \overbrace{\rho_{r}, \ldots, \rho_{r}}^{k_{r}}) .
$$

Note that $\sum_{i=1}^{r} k_{i}=N$, so that $\mathbf{k}=\left(k_{1}, \ldots, k_{r}\right)$ belongs to the set $\mathcal{P}_{N}$ of partitions of $N$ (taking order into account). Calling

$$
K_{i}=\sum_{j=1}^{i} k_{j},
$$

and dropping again the term $a^{2} E^{0}$, in the large $a$ limit Eq. (12) becomes

$$
E^{*}(\mathbf{p}, \mathbf{s}) \simeq 4 a \sum_{i=1}^{r} \rho_{i} \sum_{j=K_{i-1}+1}^{K_{i}}(N+1-2 j)=4 a \sum_{i=1}^{r} \rho_{i} l_{i},
$$

where

$$
l_{i}=k_{i}\left(N-2 K_{i}+k_{i}\right) .
$$

Since $E^{*}(\mathbf{p}, \mathbf{s})$ does not depend on the spin coordinates $\mathbf{s}$, the degeneracy associated with this eigenvalue is given by

$$
d(\mathbf{k})=\prod_{i=1}^{r}\left(\begin{array}{l}
m \\
k_{i}
\end{array}\right)
$$

so that $d(\mathbf{k})=0$ if $k_{i}>m$ for some $i$, in accordance with condition (ii). Hence,

$$
Z^{*}(4 a T) \simeq \sum_{\mathbf{k} \in \mathcal{P}_{N}} d(\mathbf{k}) \sum_{\substack{\rho_{1}>\cdots>\rho_{r} \\ k_{1} \rho_{1}+\cdots+k_{N} \rho_{r}=0}} q^{\sum_{i=1}^{r} \rho_{i} l_{i}} .
$$

Calling $\nu_{i}=\rho_{i}-\rho_{i+1} \in \mathbb{N}, i=1, \ldots, r-1$, and $\nu_{r}=\rho_{r}$, we have

$$
\sum_{i=1}^{r} \rho_{i} l_{i}=\sum_{1 \leqslant i \leqslant j \leqslant r} l_{i} \nu_{j}=\sum_{j=1}^{r} \nu_{j} N_{j}
$$

where

$$
N_{j}=\sum_{i=1}^{j} l_{i}=K_{j}\left(N-K_{j}\right)
$$

by Eq. (17). Note, in particular, that the numbers $N_{j}$ depend on $\mathbf{k}$ through the partial sums (16). Substituting (19) into (18), and taking into account that $K_{r}=N$ implies $N_{r}=0$, we obtain

$$
\begin{aligned}
Z^{*}(4 a T) & \simeq \sum_{\mathbf{k} \in \mathcal{P}_{N}} d(\mathbf{k}) \sum_{\nu_{1}, \ldots, \nu_{r-1}>0} \prod_{j=1}^{r-1} q^{N_{j} \nu_{j}} \\
& =\sum_{\mathbf{k} \in \mathcal{P}_{N}} d(\mathbf{k}) \prod_{j=1}^{r-1} \frac{q^{N_{j}}}{1-q^{N_{j}}} .
\end{aligned}
$$

Combining Eqs. (14) and (20), the partition function $Z$ can be expressed in closed form as

$$
Z(T)=\prod_{j=1}^{N-1}\left(1-q^{j(N-j)}\right) \sum_{\mathbf{k} \in \mathcal{P}_{N}} d(\mathbf{k}) \prod_{i=1}^{r-1} \frac{q^{N_{i}}}{1-q^{N_{i}}} .
$$

Note that, by definition, the partial sums $K_{i}$ are natural numbers satisfying $1 \leqslant K_{1}<\cdots<K_{r-1} \leqslant N-1$. Denoting by $K_{1}^{\prime}<\cdots<K_{N-r}^{\prime}$, the elements of the set

$$
\{1, \ldots, N-1\}-\left\{K_{1}, \ldots, K_{r-1}\right\},
$$

and setting

$$
N_{i}^{\prime}=K_{i}^{\prime}\left(N-K_{i}^{\prime}\right)
$$

we have

$$
\prod_{j=1}^{N-1}\left(1-q^{j(N-j)}\right)=\prod_{i=1}^{r-1}\left(1-q^{N_{i}}\right) \prod_{i=1}^{N-r}\left(1-q^{N_{i}^{\prime}}\right) .
$$

This identity and Eq. (21) yield the following remarkable formula for the partition function of the spin chain (2):

$$
Z(T)=\sum_{\mathbf{k} \in \mathcal{P}_{N}} \prod_{i=1}^{r}\left(\begin{array}{l}
m \\
k_{i}
\end{array}\right) q^{\Sigma_{i=1}^{r-1} N_{i}} \prod_{i=1}^{N-r}\left(1-q^{N_{i}^{\prime}}\right) .
$$

From the previous formula, it follows that the energy levels of $H$ are of the form

$$
E\left(\delta^{\prime}\right)=\sum_{j=1}^{N-1} \delta_{j}^{\prime} j(N-j),
$$

where $\delta_{j}^{\prime}=1$ if $j$ is one of the partial sums $\widetilde{K}_{i}$ corresponding to a partition $\left(\widetilde{k}_{1}, \ldots, \widetilde{k}_{r}\right) \in \mathcal{P}_{N}$ with $\widetilde{k}_{l} \leqslant m$ for all $l$ [by condition (ii)], and $\delta_{j}^{\prime}=0$ otherwise. In order to relate Eq. (23) with the known expression (1) for the energies of the original HS Hamiltonian, we need to evaluate the maximum energy $E_{\max }$. From Eq. (3), we have

$$
\begin{aligned}
E_{\max } & =\sum_{j=1}^{N-1}(N-j) \csc ^{2}\left(\frac{j \pi}{N}\right)=\sum_{j=1}^{N-1} j \csc ^{2}\left(\frac{(N-j) \pi}{N}\right) \\
& =\sum_{j=1}^{N-1} j \csc ^{2}\left(\frac{j \pi}{N}\right) .
\end{aligned}
$$

Hence 


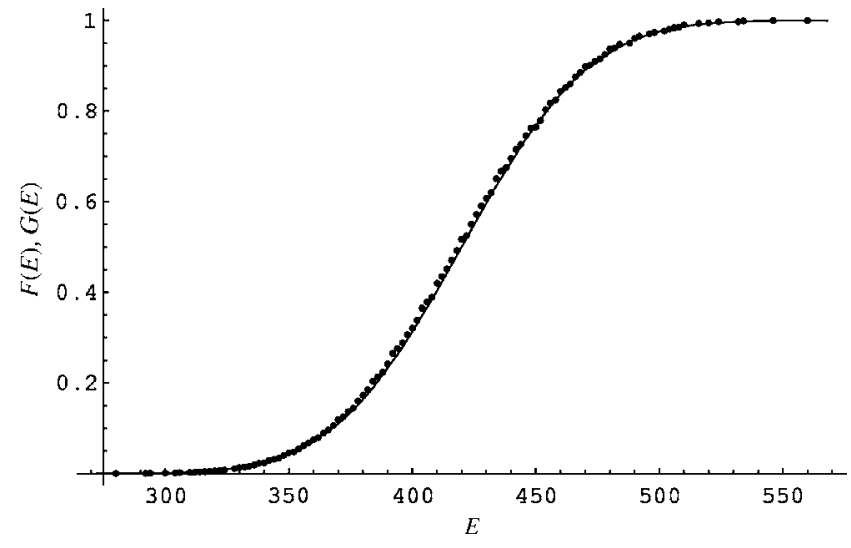

FIG. 1. Cumulative distribution functions $F(E)$ (at its discontinuity points) and $G(E)$ (continuous line) for $N=15$ and $m=2$.

$$
E_{\max }=\frac{N}{2} \sum_{j=1}^{N-1} \csc ^{2}\left(\frac{j \pi}{N}\right)=\frac{N}{6}\left(N^{2}-1\right),
$$

where the last sum is evaluated in Ref. 25. Since the RHS of (24) coincides with the sum $\sum_{j=1}^{N-1} j(N-j)$, Eq. (23) implies Eq. (1) with $\delta_{j}=1-\delta_{j}^{\prime}$. In particular, from the latter relation between $\delta$ and $\delta^{\prime}$ it follows that $\delta$ is a motif with no more than $m-1$ consecutive 1 's.

\section{LEVEL DENSITY AND SPACINGS DISTRIBUTION}

The RHS of Eq. (22) is a polynomial in $q$ whose evaluation with a symbolic algebra package is straightforward once $N$ and $m$ are fixed. In this way, we have been able to compute the spectrum of the chain (2) for relatively large values of $N$ and $m$, for which the usual motif approach becomes inefficient due to the difficulty of computing the degeneracies. From the analysis of the spectral data thus obtained, one can infer several global properties of the spectrum that we shall now discuss. In the first place, it is apparent that for $N \gg 1$, the level density is Gaussian to a very high degree of accuracy, as in the HS spin chain of $B C_{N}$ type studied in Ref. 22. In other words, for large $N$ the cumulative level density

$$
F(E)=m^{-N} \sum_{i ; E_{i} \leqslant E} d_{i}
$$

is approximately given by

$$
G(E)=\frac{1}{2}\left[1+\operatorname{erf}\left(\frac{E-\mu}{\sqrt{2} \sigma}\right)\right],
$$

where $d_{i}$ is the degeneracy of the energy $E_{i}$, and $\mu$ and $\sigma$ are, respectively, the mean and the standard deviation of the energy. This can already be seen, for instance, in the case $N$ $=15$ and $m=2$ presented in Fig. 1. The agreement between $F$ and $G$ rapidly improves as $N$ and/or $m$ grow, e.g., for $m=2$, the mean square error decreases from $5.2 \times 10^{-5}$ for $N=15$ to $5.6 \times 10^{-6}$ for $N=20$, or from $2.6 \times 10^{-5}$ for $N=15$ to 2.6 $\times 10^{-6}$ for $N=20$ when $m=3$.

Since, by the previous discussion, for large $N$, the level density is characterized by $\mu$ and $\sigma$ through the Gaussian law, it is of interest to compute these parameters in closed form as functions of $N$ and $m$. In the first place, using the identity $\operatorname{tr} S_{i j}=m^{N-1}$ and Eqs. (3) and (24), we obtain

$$
\mu=\frac{\operatorname{tr} H}{m^{N}}=\frac{m+1}{2 m} \sum_{i<j} \csc ^{2}\left(\xi_{i}-\xi_{j}\right)=\frac{m+1}{12 m} N\left(N^{2}-1\right) .
$$

Similarly, the formula

$$
\operatorname{tr}\left(S_{i j} S_{k l}\right)=m^{N-2+2 \delta_{i k} \delta_{j l}+2 \delta_{i l} \delta_{j k}}
$$

yields

$$
\begin{aligned}
\sigma^{2} & =\frac{\operatorname{tr}\left(H^{2}\right)}{m^{N}}-\frac{(\operatorname{tr} H)^{2}}{m^{2 N}}=\frac{m^{2}-1}{4 m^{2}} \sum_{i<j} \csc ^{4}\left(\xi_{i}-\xi_{j}\right) \\
& =\frac{\left(m^{2}-1\right) N}{8 m^{2}} \sum_{j=1}^{N-1} \csc ^{4} \xi_{j}=\frac{m^{2}-1}{360 m^{2}} N\left(N^{2}-1\right)\left(N^{2}+11\right)
\end{aligned}
$$

(cf. Ref. 25 for the last equality).

The level density is also Gaussian as $N \rightarrow \infty$ for the socalled "embedded Gaussian orthogonal ensemble"26 (EGOE) in random matrix theory. Note, however, that in the EGOE this property is valid provided that the number of oneparticle states tends to infinity faster than $N$. This additional condition clearly does not hold in our case, since the number of one-particle states (i.e., $m$ ) is fixed. Another characteristic feature of the EGOE is the fact that the nearest-neighbor spacing distribution $p(s)$ is approximately given by Wigner's law

$$
p(s)=(\pi / 2) s \exp \left(-\pi s^{2} / 4\right),
$$

as for the classical Gaussian orthogonal ensemble. ${ }^{27}$ On the other hand, since the HS spin chain is integrable, one would expect that its nearest-neighbor spacing distribution obey Poisson's law $p(s)=\mathrm{e}^{-s}$, according to the conjecture of Berry and Tabor for a generic integrable model. ${ }^{28}$ This conjecture has been verified for a variety of integrable many-body problems, such as the Heisenberg chain, the $t-J$ model, the Hubbard model, ${ }^{29}$ and the chiral Potts model. ${ }^{30}$ One of the main results of this paper is the fact that the nearest-neighbor spacing distribution of the HS chain deviates substantially from both Wigner's and Poisson's laws.

In order to correctly take into account the effect of the local level density in the study of $p(s)$, one must first apply to the "raw" spectrum the so-called unfolding mapping. ${ }^{31}$ This mapping is defined by decomposing the cumulative level density $F(E)$ as the sum of a fluctuating part $F_{\mathrm{fl}}(E)$ and a continuous part $\xi(E)$, which is then used to transform each energy $E_{i}, i=1, \ldots, n$, into an unfolded energy $\xi_{i}=\xi\left(E_{i}\right)$. The function $p(s)$ is defined as the density of the normalized spacings $s_{i}=\left(\xi_{i+1}-\xi_{i}\right) / \Delta$, where $\Delta=\left(\xi_{n}-\xi_{1}\right) /(n-1)$ is the mean spacing of the unfolded energies. By the previous discussion, in our case we can take the unfolding mapping $\xi(E)$ as the cumulative Gaussian distribution $G(E)$ with parameters $\mu$ and $\sigma$ given by the previous formulas. As for the level density, to compare the discrete distribution function $p(s)$ with a continuous distribution it is more convenient to work with the cumulative spacing distribution $P(s)$ $=\int_{0}^{s} p(x) d x$. Our computations for a wide range of values of 


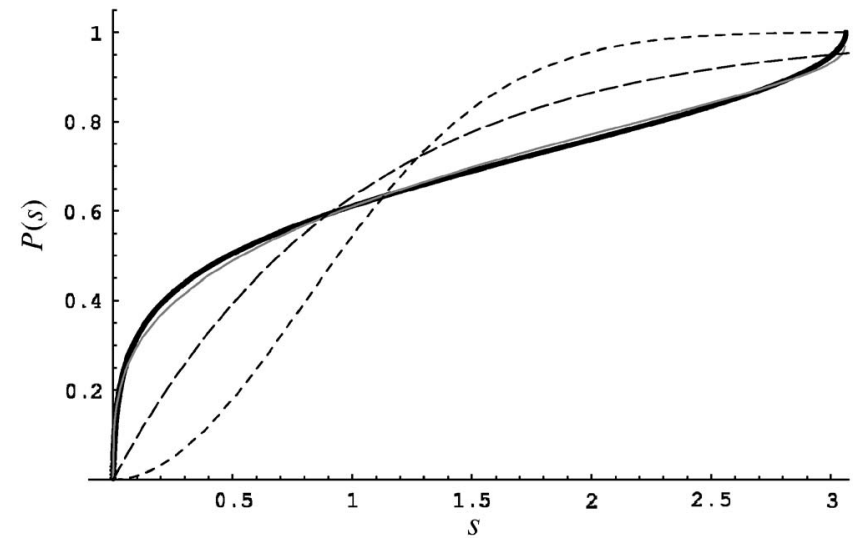

FIG. 2. Cumulative spacing distribution $P(s)$ and its approximation $\widetilde{P}(s)$ (gray line) for $N=26$ and $m=2$. For convenience, we have also represented Poisson's (long dashes) and Wigner's (short dashes) cumulative distributions.

$N$ and $m$ show that $P(s)$ is essentially different from either Poisson's or Wigner's law, since its slope tends to infinity both as $s \rightarrow 0$ and $s \rightarrow s_{\max }$, where $s_{\max }$ is the largest spacing. In fact, it turns out that in all cases $P(s)$ is well approximated by a cumulative distribution of the simple form

$$
\widetilde{P}(s)=t^{\alpha}\left[1-\gamma(1-t)^{\beta}\right],
$$

where $t=s / s_{\max }$ and $0<\alpha, \beta<1$. The parameter $\gamma$ is fixed by requiring that the average spacing be equal to 1 , with the result

$$
\gamma=\left(\frac{1}{s_{\max }}-\frac{\alpha}{\alpha+1}\right) / B(\alpha+1, \beta+1)
$$

where $B$ is Euler's beta function. For instance, for $N=26$ and $m=2$ the largest spacing is $s_{\max }=3.06$, and the best leastsquares fit parameters $\alpha$ and $\beta$ are, respectively, 0.31 and 0.23 , with a mean square error of $4.1 \times 10^{-4}$ (see Fig. 2).

For a fixed value of $m$, the parameters $\alpha, \beta$, and $s_{\max }$ vary smoothly with $N \gtrsim 15$, provided that $N$ has a fixed parity. ${ }^{35}$

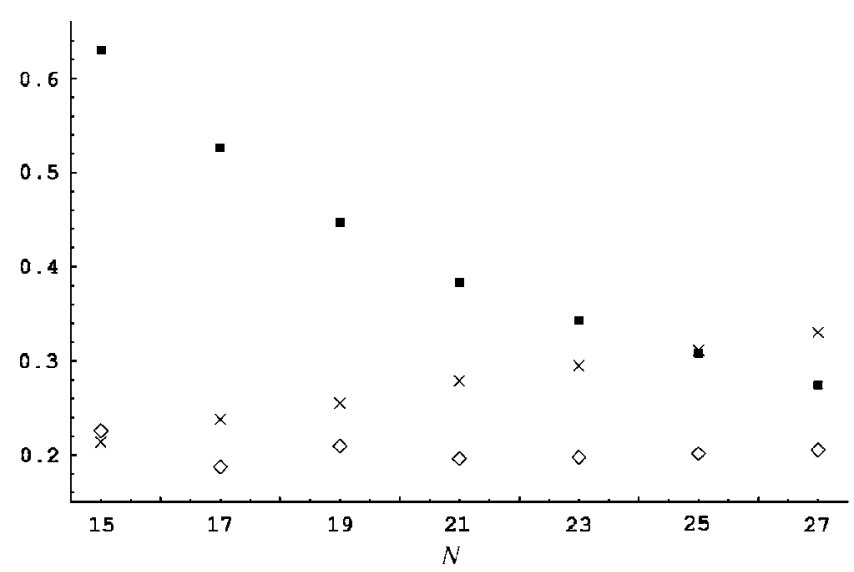

FIG. 3. Values of $\alpha$ (box), $\beta$ (rhombus), and $s_{\max } / 10$ (cross) for $m=2$ and odd $N$.

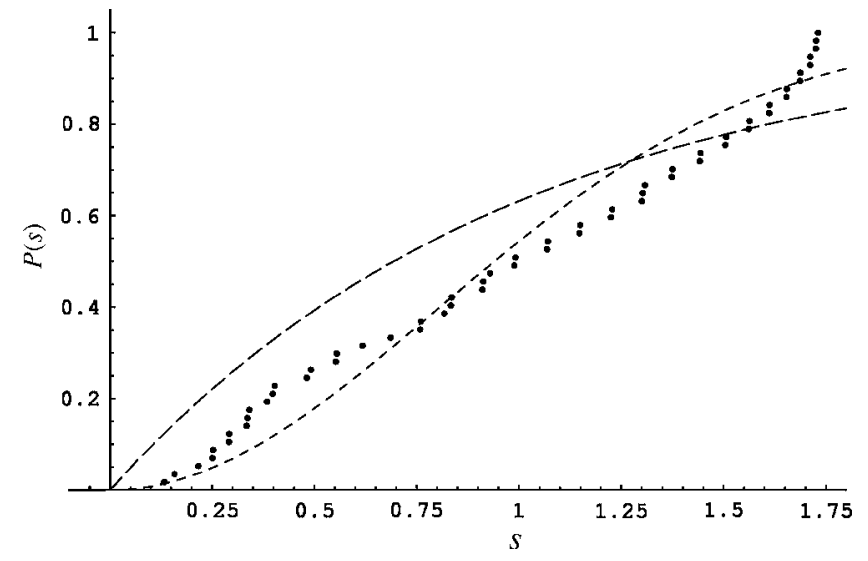

FIG. 4. Cumulative spacing distribution $P(s)$ (solid dots) for states with zero total spin and odd parity when $N=13$ and $m=2$. For comparison purposes, we have represented Poisson's (long dashes) and Wigner's (short dashes) cumulative distributions.

For instance, in Fig. 3, we plot these parameters for $m=2$ and odd $N$ running from 15 to 27 (the plot for even $N$ is very similar). In all cases, the fit of the distribution (25) to the data is quite good, the mean square error never exceeding $7.4 \times 10^{-4}$. We have performed a similar analysis for $m=3$ and $15 \leqslant N \leqslant 22$, obtaining totally analogous results.

The divergence of the nearest-neighbor spacing distribution $p(s)$ for small $s$ is probably related to the flatness of the tail of the Gaussian distribution. It could also be argued that, since the Haldane-Shastry chain is completely integrable, the full spectrum is a superposition of the spectra of the Hamiltonian restricted to subspaces of common eigenfunctions of a suitable family of commuting first integrals. It is well known, in this respect, that a superposition of a large number of unrelated spectra leads to a sharp increase in the number of very small spacings. ${ }^{32}$ On the other hand, we do not have a clear explanation of the fact that $p(s)$ also diverges when $s$ approaches the largest spacing $s_{\max }$. This fact, which certainly deserves further study, could be a characteristic property of all spin chains of Haldane-Shastry type.

Our results also imply that Berry and Tabor's conjecture does not hold for the HS spin chain, even if we restrict ourselves to a subspace of the whole Hilbert space with welldefined quantum numbers. Indeed, the nearest-neighbor spacing distribution of the superposition of even a small number of spectra with Poisson-distributed spacings must also be of Poisson type. ${ }^{32}$ As an illustration of these assertions, we present in Fig. 4 a plot of the cumulative spacing distribution corresponding to the restriction of the Hamiltonian (2) to the subspace with zero total spin and odd parity for $N=13$ and $m=2$, obtained by a numerical computation of the spectrum of $H$ restricted to this subspace. It is apparent from this plot that $P(s)$ is neither Poissonian nor of Wigner type, and that it is well approximated by a function of the form (25) for spacings $s \gtrsim 0.25$. It is also clearly noticeable that $p(s)$ tends to infinity as $s$ approaches the maximum spacing $s_{\max } \simeq 1.73$.

The non-Poissonian behavior of the spacing distribution could in principle be due to finite-size effects. ${ }^{33}$ Although 
this possibility should be explored in more detail, our data clearly show that the cumulative spacing distribution $P(s)$ is of the form (25) for a wide range of values of $N \leqslant 28$.

Note, finally, that an interesting integrable model not obeying the Berry-Tabor conjecture has been recently constructed in Ref. 34. In contrast with the HS spin chain, the latter model is a nongeneric element of a class depending on a large number of parameters and involves many-body interactions.

\section{ACKNOWLEDGMENTS}

This work was partially supported by the Spanish DGI under Grant No. BFM2002-02646. The authors would like to thank J. Retamosa for several helpful discussions.
*Electronic address: ffinkel@ fis.ucm.es

Corresponding author. Electronic address: artemio@ fis.ucm.es

${ }^{1}$ F. D. M. Haldane, Phys. Rev. Lett. 60, 635 (1988).

${ }^{2}$ B. S. Shastry, Phys. Rev. Lett. 60, 639 (1988).

${ }^{3}$ M. C. Gutzwiller, Phys. Rev. Lett. 10, 159 (1963).

${ }^{4}$ F. Gebhard and D. Vollhardt, Phys. Rev. Lett. 59, 1472 (1987).

${ }^{5}$ C. Gros, R. Joynt, and T. M. Rice, Phys. Rev. B 36, 381 (1987).

${ }^{6}$ P. W. Anderson, G. Baskaran, Z. Zou, and T. Hsu, Phys. Rev. Lett. 58, 2790 (1987).

${ }^{7}$ M. Fowler and J. A. Minahan, Phys. Rev. Lett. 70, 2325 (1993).

${ }^{8}$ F. D. M. Haldane, Phys. Rev. Lett. 66, 1529 (1991).

${ }^{9}$ N. Kawakami, Phys. Rev. B 46, 1005 (1992).

${ }^{10}$ Z. N. C. Ha and F. D. M. Haldane, Phys. Rev. B 47, 12459 (1993)

${ }^{11}$ F. D. M. Haldane, Phys. Rev. Lett. 67, 937 (1991).

${ }^{12}$ F. D. M. Haldane, Z. N. C. Ha, J. C. Talstra, D. Bernard, and V. Pasquier, Phys. Rev. Lett. 69, 2021 (1992).

${ }^{13}$ D. Bernard, M. Gaudin, F. D. M. Haldane, and V. Pasquier, J. Phys. A 26, 5219 (1993).

${ }^{14}$ C. F. Dunkl, Trans. Am. Math. Soc. 311, 167 (1989).

${ }^{15}$ A. P. Polychronakos, Phys. Rev. Lett. 69, 703 (1992).

${ }^{16}$ B. Sutherland, Phys. Rev. A 4, 2019 (1971).

${ }^{17}$ B. Sutherland, Phys. Rev. A 5, 1372 (1972).

${ }^{18}$ K. Hikami and B. Basu-Mallick, Nucl. Phys. B 566, 511 (2000).

${ }^{19}$ K. Hikami and M. Wadati, J. Math. Phys. 44, 3569 (2003).
${ }^{20}$ H. Frahm, J. Phys. A 26, L473 (1993).

${ }^{21}$ A. P. Polychronakos, Phys. Rev. Lett. 70, 2329 (1993).

${ }^{22}$ A. Enciso, F. Finkel, A. González-López, and M. A. Rodríguez, Nucl. Phys. B 707, 553 (2005).

${ }^{23}$ A. P. Polychronakos, Nucl. Phys. B 419, 553 (1994).

${ }^{24}$ B. Basu-Mallick, Nucl. Phys. B 482, 713 (1996).

${ }^{25}$ F. Calogero and A. M. Perelomov, Commun. Math. Phys. 59, 109 (1978).

${ }^{26}$ K. K. Mon and J. B. French, Ann. Phys. 95, 90 (1975).

${ }^{27}$ V. K. B. Kota, Phys. Rep. 347, 223 (2001).

${ }^{28}$ M. V. Berry and M. Tabor, Proc. R. Soc. London 356, 375 (1977).

${ }^{29}$ D. Poilblanc, T. Ziman, J. Bellissard, F. Mila, and J. Montambaux, Europhys. Lett. 22, 537 (1993).

${ }^{30}$ J-C. A. d'Auriac, J-M. Maillard, and C. M. Viallet, J. Phys. A 35, 4801 (2002).

${ }^{31}$ F. Haake, Quantum Signatures of Chaos, 2nd ed. (SpringerVerlag, Berlin, 2001).

${ }^{32}$ N. Rosenzweig and C. E. Porter, Phys. Rev. 120, 1698 (1960).

${ }^{33}$ K. Kudo and T. Deguchi, J. Phys. Soc. Jpn. 74, 1992 (2005).

${ }^{34}$ A. Relaño, J. Dukelsky, J. M. G. Gómez, and J. Retamosa, Phys. Rev. E 70, 026208(5) (2004).

${ }^{35}$ Our computations show that the number of levels, and hence of different spacings, increases monotonically with $N$ of a fixed parity, but decreases when $N$ jumps from $2 j$ to $2 j+1$. 